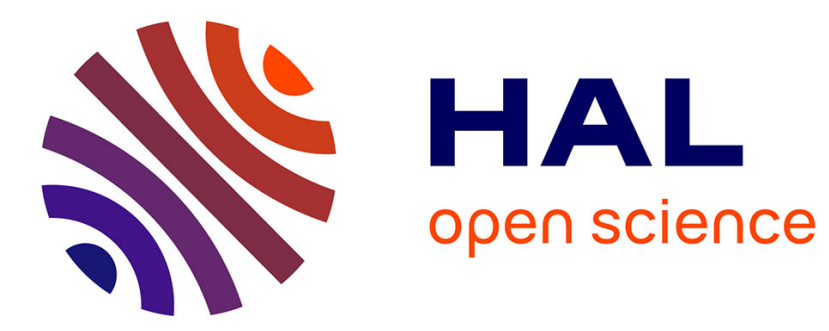

\title{
Complications respiratoires postopératoires
}

Antoine Nau, Mohamed Hadj, Mathieu Raux

\section{To cite this version:}

Antoine Nau, Mohamed Hadj, Mathieu Raux. Complications respiratoires postopératoires. Praticien en Anesthésie Réanimation, 2016, 10.1016/j.pratan.2016.02.006 . hal-01288616

\section{HAL Id: hal-01288616 https: / hal.sorbonne-universite.fr/hal-01288616}

Submitted on 15 Mar 2016

HAL is a multi-disciplinary open access archive for the deposit and dissemination of scientific research documents, whether they are published or not. The documents may come from teaching and research institutions in France or abroad, or from public or private research centers.
L'archive ouverte pluridisciplinaire HAL, est destinée au dépôt et à la diffusion de documents scientifiques de niveau recherche, publiés ou non, émanant des établissements d'enseignement et de recherche français ou étrangers, des laboratoires publics ou privés. 


\section{Complications respiratoires post opératoires}

Antoine NAU (1), Mohamed HADJ, (1), Mathieu RAUX (2,3)

(1) Hôpital d'instruction des armées Percy, Département d'Anesthésie Réanimation, Clamart, France

(2) AP-HP, Groupe Hospitalier Pitié-Salpêtrière Charles Foix, Département d'Anesthésie Réanimation, F-75013, Paris, France

(3) Sorbonne Universités, UPMC Univ Paris 06, INSERM, UMRS1158 Neurophysiologie respiratoire expérimentale et clinique, Paris, France

\section{Correspondance:}

Dr. Mathieu RAUX

Salle de Surveillance Post-Interventionnelle et d'Accueil des Polytraumatisés

Département d'Anesthésie Réanimation

Groupe hospitalier Pitié-Salpêtrière

47-83 Boulevard de l'Hôpital

75651 Paris Cedex 13, France

Tel : +33142177329

Fax : +33 142177388

Email : mathieu.raux@psl.aphp.fr 


\section{Introduction}

Les complications respiratoires postopératoires (CRP) sont d'expression variées, allant de l'atélectasie avec désaturation modérée à la détresse respiratoire nécessitant une réintubation (1) . C'est un problème important, probablement sous-estimé, dont l'incidence et la morbidité sont au moins égales à celles des complications cardio-vasculaires post opératoires (2).

L'anesthésiste-réanimateur doit désormais intégrer ce risque dans sa prise en charge péri anesthésique, ce qui nécessite de bien connaître l'étendue du problème, de comprendre les mécanismes de survenue, de savoir identifier les patients à risque, et de mettre en place des moyens de prévention en pré, per, et post-opératoire.

\section{Incidence des complications respiratoires post-opératoires}

L'incidence des CRP dépend de la définition que l'on en donne. Si on les définit comme l'apparition d'un Acute Lung Injury ou d'un Syndrome de Détresse Respiratoire Aiguë post chirurgie à haut risque, on retrouve une incidence de $2.6 \%$ (3). Une définition plus restrictive (nécessité de ventilation mécanique $>48$ heures) montre une incidence des CRP de $3,4 \%$ sur tout type de chirurgie, avec des incidences notablement plus élevées (entre 5 et 10\%) en chirurgie abdominale, thoracique et vasculaire (4). Enfin, si on utilise une définition beaucoup plus large (infection respiratoire, défaillance respiratoire définie par une saturation en oxygène en air ambiant inférieure à $90 \%$ ou une pression artérielle partielle en oxygène inférieure à $60 \mathrm{mmHg}$, épanchement pleural, atélectasie, pneumothorax, bronchospasme...) l'incidence est plus élevée et atteint 5\% de l'ensemble des chirurgies, avec des pics à $39.6 \%$ en chirurgie cardiaque, $31.4 \%$ en chirurgie thoracique et $7.4 \%$ en chirurgie digestive (1).

Si l'on compare l'incidence des CRP et celle des complications cardiovasculaires en chirurgie non cardiaque, on aboutit à des chiffres relativement semblables (2). Il semble même que la survenue de CRP soit plus prédictive de la mortalité à long terme que les complications cardio-vasculaires (5). 
Ainsi, les CRP représentent un véritable problème, probablement sous-estimé, alors que le risque de complications cardio-vasculaires est bien connu et intégré depuis longtemps à la prise en charge anesthésique.

\section{Mécanismes de survenue des CRP}

Les trois grands déterminants de survenue des CRP sont liés à l'anesthésie, à la chirurgie, et au terrain.

\subsection{L'anesthésie}

Les produits d'anesthésie entraînent une diminution du tonus musculaire avec augmentation des forces rétractiles du parenchyme pulmonaire. Il en résulte une diminution de la capacité résiduelle fonctionnelle (CRF) de plus de 20\% (6). Cette diminution est également responsable de l'apparition d'atélectasies dès que la CRF passe en dessous du volume de fermeture $(7,8)$ avec pour conséquence une majoration du shunt physiologique et baisse du rapport ventilation/perfusion. Enfin, la baisse du tonus musculaire entraîne la fermeture des petites voies aériennes, ce qui majore encore le shunt dans certaines zones (7). Ces effets sont exacerbés en décubitus dorsal et chez l'obèse (9).

D’autres mécanismes ont été proposés pour expliquer la diminution du rapport ventilation/perfusion, au premier rang desquels figure la compression des régions inférieures du poumon par l'abdomen, due à la perte de la rigidité diaphragmatique (8). On retrouve par ailleurs la résorption des gaz notamment en cas d'inhalation d'oxygène pur (10), ainsi que les altérations du surfactant (11).

Enfin, en post-opératoire, deux mécanismes liés à l'anesthésie peuvent entraîner des complications respiratoires. D'une part, la sédation résiduelle peut être responsable d'une obstruction de la filière pharyngée (12) et augmenter ainsi le risque d'inhalation par relaxation du sphincter supérieur de l'œsophage (13). D'autre part, toute curarisation résiduelle expose à un risque de dépression respiratoire par perte de coordination des muscles intercostaux et parésie diaphragmatique, ainsi qu'à un risque prolongé d'inhalation par dysfonction de la filière laryngo-pharyngée (14). 
Globalement, 1'anesthésie générale contribue, à elle seule, au doublement du risque de complications respiratoires post chirurgicales comparativement à une anesthésie locorégionale (2).

\subsection{La chirurgie}

Le geste chirurgical en lui-même induit une altération de la fonction respiratoire, par différents mécanismes.

Le premier d'entre eux est le traumatisme musculaire, par lésion chirurgicale du diaphragme ou des muscles intercostaux ou abdominaux. Ainsi, le type d'incision semble jouer un rôle important. Les incisions transverses entraînent moins de CRP que les incisions médianes (15). La chirurgie par cœlioscopie entraîne une moindre altération de la fonction respiratoire que la laparotomie $(16,17)$.

Le second mécanisme chirurgical à l'origine de CRP est la douleur, qui contribue à restreindre la course diaphragmatique. Enfin, la stimulation péritonéale entraîne une inhibition de la commande phrénique, via des afférences viscérales situées au niveau du mésentère et de nombreux organes intra-abdominaux $(18,19)$. A ces titres, l'altération de la fonction diaphragmatique est d'autant plus marquée que la chirurgie est proche du diaphragme (chirurgie aortique, thoracique, sus-mésocolique) (2).

Aux conséquences directes de la chirurgie, viennent s'ajouter d'autres mécanismes responsables d'une altération parenchymateuse, tels l'inflammation, le sepsis, ou le Transfusion Related Acute Lung Injury (2).

Cette dysfonction respiratoire est prévisible et atteint son paroxysme dans les 48 heures postopératoires (20). Il apparaît donc important d'optimiser la programmation de l'acte chirurgical de sorte que le nadir de la fonction ventilatoire corresponde à un jour « ouvrable » lorsque le patient est à risque élevé de CRP. Il est donc illogique d'optimiser le patient à haut risque tout en programmant sa chirurgie en fin de semaine.

\subsection{Le terrain du patient}

Dans l'étude de Smetana, les facteurs de risque de CRP les plus importants liés au patient étaient l'âge, un score $\mathrm{ASA} \geq 2$, la présence d'une insuffisance cardiaque chronique, d'une limitation fonctionnelle pré opératoire (définie par une dépendance dans les actes de la vie quotidienne), d'une bronchopneumopathie chronique obstructive (2). Arozullah et coll. 
retrouvaient en 2001 globalement les mêmes facteurs pour prédire la survenue d'une pneumopathie post opératoire (21). Canet et coll. y ajoutaient en 2010 une saturation en oxygène pré opératoire $\leq 90 \%$, la notion d'infection respiratoire dans le mois précédent, et une anémie pré opératoire (1). L’ensemble de ces éléments sont évaluables en consultation d'anesthésie, sans recourir à d'autres examens complémentaires.

Ces facteurs de risque liés au patient s'intègrent dans des scores prédictifs de risque de CRP détaillés ultérieurement.

\section{Identification des patients à risque :}

\subsection{Scores de risque}

De la même façon qu'il existe depuis longtemps des scores de risque de complications cardiovasculaires post opératoires en chirurgie non cardiaque (22), dont le plus connu et le plus utilisé est le score de Lee (23), plusieurs auteurs ont tenté de développer des scores prédictifs de CRP.

L'un des premiers scores de prédiction de pneumopathie post opératoire, « postoperative pneumoniae risk index », identifie des facteurs de risque liés au patient et à la chirurgie, pondérés selon leur rapport de cote. Selon le nombre de points, les patients sont classés en 5 groupes, avec une probabilité de pneumopathie post opératoire allant de $0.24 \%$ à $15.3 \%$ (21). Le nombre d'items est important (quatorze), ce qui rend l'utilisation de ce score difficile en routine.

D'autres scores se sont plus spécifiquement attachés à la prédiction du risque de réintubation (24). Tous ces scores ont été mis au point sur des cohortes de patients spécifiques dans un centre donné et posent donc le problème de leur validité externe.

Plus récemment, Canet et al ont décrit le score ARISCAT ( Assess Respiratory Risk in Surgical Patients in Catalonia ») (1). Leur définition des CRP est plus large et pragmatique, et leur score comporte moins d'items (sept). Il permet de classer les patients en trois groupes (Bas risque : 1.6\% de CRP; Risque intermédiaire 13.3\%, Haut risque 42.1\%) et prend en compte à la fois les facteurs liés au patient et à la chirurgie. Ce score a été validé dans une large cohorte européenne (25) et semble donc être à l'heure actuelle le plus intéressant et le plus utilisable en pratique clinique.

Il est important de noter que dans la plupart des scores et en particulier dans ARISCAT, les facteurs liés à la chirurgie semblent plus importants que ceux liés au patient. 


\subsection{Examens complémentaires}

Il peut sembler tentant de recourir aux examens complémentaires pour identifier les patients à risque de complications respiratoires post opératoires. Mais la radiographie pulmonaire, les gaz du sang, tout comme les épreuves fonctionnelles respiratoires pré opératoires ont de faibles valeurs prédictives positives et négatives de CRP en chirurgie non cardio-thoracique. Ils ne sont pas plus informatifs que la clinique (2). La plupart de ces examens ne sont d'ailleurs plus recommandés en pré opératoire de manière systématique (26).

Parmi les examens de laboratoire qui pourraient être utiles pour stratifier le risque, il semble que seul un taux d'albumine $<35 \mathrm{~g} / 1$ soit associé à un sur-risque significatif de CRP (2). Le dosage de l'albuminémie est d'ailleurs recommandé chez les patients à risque dans les guidelines américaines de prévention des CRP (27).

\section{Prévention de la survenue des CRP}

Une fois le risque identifié, il convient de mettre en œuvre les moyens permettant de le réduire.

\subsection{Prévention pré opératoire}

Le tabac, même s'il ne fait pas partie des facteurs de risque de CRP les plus importants, majore tout de même ce risque. L'arrêt du tabac est donc recommandé, même si les niveaux de preuve sont bas (28). Il se fera au mieux 2 mois avant la chirurgie et sera maintenu pendant la phase de cicatrisation. Il faut noter que l'arrêt du tabac, même $24 \mathrm{~h}$ avant une chirurgie reste recommandé (28). Le risque avancé d'encombrement bronchique lié à une broncho-sécrétion importante à son arrêt reste moindre que le bénéfice global observé.

La spirométrie incitative permet de diminuer de manière significative la survenue des CRP et particulièrement des pneumopathies post opératoires en chirurgie cardiaque. Elle n'est toutefois pas facile à mettre en œuvre et nécessite une parfaite compréhension et la coopération du patient.

La kinésithérapie pré opératoire est intéressante chez les patients présentant une bronchopneumopathie chronique obstructive, car elle améliore leur capacité fonctionnelle pré opératoire (29). 
La ventilation non invasive (VNI) préopératoire à deux niveaux de pression, à la fréquence de cinq fois une heure par jour, en chirurgie thoracique, associée à la kinésithérapie respiratoire et à des aérosols broncho-dilatateurs montre une amélioration des gaz du sang et des épreuves fonctionnelles respiratoires post opératoires immédiates (30).

\subsection{Prévention per opératoire}

L'anesthésie générale en elle-même étant un facteur de risque, il convient de favoriser l'anesthésie loco régionale chez les patients les plus à risque, en l'absence de contreindication et lorsque la chirurgie le permet. En outre, le monitorage de la curarisation est bien sûr indispensable pour éviter le risque de curarisation résiduelle.

Un des principaux facteurs sur lequel l'anesthésiste peut agir est la limitation de l'agression pulmonaire. La nécessité de mise en œuvre d'une ventilation protectrice dans le SDRA est bien documentée et acceptée par tous $(31,32)$. Sur des poumons présumés sains, cette pratique était plus controversée, les résultats étant contradictoires. En effet, la ventilation protectrice était accusée d'entraîner une hypoventilation alvéolaire et l'apparition d'atélectasies. Ainsi, en pratique courante, seule une faible proportion de patients recevait une ventilation protectrice, des volumes courants élevés et l'absence de PEEP étant fréquents $(33,34)$.

On peut considérer que même chez un patient à poumons sains, la réponse inflammatoire, l'apparition d'atélectasies et la ventilation mécanique elle-même sont à risque d'entraîner des lésions alvéolaires. La pratique d'une ventilation protectrice pourrait alors se révéler intéressante. L'étude IMPROVE, qui comparait chez des patients de chirurgie abdominale réglée une stratégie de ventilation protectrice (volume courant 6-8 ml/kg, pression expiratoire positive 6-8 $\mathrm{cmH} 20$, et manœuvres de recrutement) à une ventilation conventionnelle (volume courant $8-12 \mathrm{ml} / \mathrm{kg}$, pas de pression expiratoire positive ni de manœuvre de recrutement) retrouvait une baisse significative des complications respiratoires (pneumopathie, nécessité de VNI post opératoire ou de réintubation) et extra respiratoires dans le groupe ventilation protectrice (35).

A la lumière de ces résultats, on peut aujourd'hui recommander l'utilisation d'une ventilation protectrice, en particulier chez les patients les plus à risque de complications postopératoires. 
Les modalités proposées sont les suivantes :

- Volume courant $(\mathrm{Vt})=6-8 \mathrm{ml} / \mathrm{kg}$ de poids idéal. L'utilisation du poids idéal pour le calcul du volume courant est importante pour éviter le risque d'insuffler un Vt beaucoup trop important, en particulier chez l'obèse.

- Pression de plateau $<30 \mathrm{cmH} 20$

- $\quad$ PEEP 6-8 cmH20

- Manœuvres de recrutement: application d'une pression positive de $+30 \mathrm{cmH} 20$ pendant 30 secondes après l'intubation, puis toutes les 30 minutes, ou en cas de désaturation. On sera vigilant devant le risque de baisse du débit cardiaque induit par ces manœuvres de recrutement chez les patients instables hémodynamiquement.

\subsection{Prévention post opératoire}

\subsubsection{L'analgésie post opératoire :}

La péridurale thoracique (APD) est largement utilisée pour assurer l'analgésie post opératoire en chirurgie abdominale ou thoracique majeure. Popping, dans une méta analyse de 2008 reprenant des études publiées entre 1971 et 2006, montrait un bénéfice clair de l'analgésie péridurale en post opératoire de chirurgie abdominale ou thoracique, avec une réduction du risque de pneumopathie post opératoire et une amélioration des variables spirométriques et gazométriques à $24 \mathrm{~h}$ (36). Cependant, on observe une baisse de ces effets bénéfiques avec le temps, probablement due à l'amélioration des moyens de l'analgésie systémique (analgésie morphinique contrôlée par le patient, développement du concept d'analgésie multimodale). Cela fait donc relativiser l'intérêt de la péridurale thoracique, d'autant plus qu'il s'agit d'une technique invasive, non dénuée de risques (abcès, hématome, hypotension per opératoire, rétention aigue d'urines postopératoire), et qui nécessite le plus 
souvent une surveillance spécifique en secteur de surveillance continue. En outre, elle peut être de réalisation difficile, voire contre-indiquée chez des patients sous antiagrégants plaquettaires ou anticoagulants. Il convient donc pour chaque patient, au sein de chaque structure, de bien peser le rapport bénéfice/risque.

D'autres techniques d'analgésie locorégionale peuvent être utilisées. Ainsi, l'infiltration cicatricielle est associée à une moindre dysfonction diaphragmatique (37). Le bénéfice ventilatoire du Transverse Abdominal Plan (ou TAP) bloc, ainsi que du bloc paravertébral doit être évalué.

\subsubsection{Expansion pulmonaire.}

L'expansion pulmonaire post opératoire vise à augmenter les forces expansives du poumon, ou à augmenter le recrutement alvéolaire. Dans le premier cas, on utilise des modalités volitionnelles: spirométrie incitative, kinésithérapie respiratoire. La spirométrie incitative est une technique visant à encourager le patient à effectuer des inspirations maximales. A cette fin, on utilise un appareil (spiromètre incitatif) qui donne au patient un retour visuel lié au débit inspiratoire produit. Lawrence et al. , dans une revue systématique de 2006, rapportent des effets bénéfiques sur les CRP de la spirométrie incitative et des exercices ventilatoires en chirurgie non cardiothoracique (38). Néanmoins, la revue de Freitas en 2012 ne retrouvait pas ce bénéfice chez des patients de chirurgie cardiaque, en particulier du fait du faible nombre d'essais cliniques (39). Ce sont des techniques facilement réalisables, peu onéreuses, sans effets secondaires, mais qui nécessitent néanmoins des dispositifs spécifiques (spiromètre) et la coopération du patient.

Dans le second cas, on utilise des techniques ne faisant pas appel à la coopération volontaire du patient, mais amplifiant les effets de son comportement ventilatoire, d'où leur nom de techniques comportementales. La principale technique comportementale est la VNI. Cette dernière peut prendre deux formes, selon le niveau de pressurisation choisi. Lorsqu'elle ne délivre qu'un seul niveau de pression tout au long du cycle ventilatoire, la VNI porte le nom de Pression Positive Continue (PPC ou Continuous Positive Airway Pressure - CPAP). Une méta analyse de 2008, reprenant des études dans lesquelles la PPC était utilisée en prophylactique et en curatif (devant une insuffisance respiratoire aiguë post opératoire), retrouvait une diminution du taux de complication respiratoire en post opératoire de chirurgie abdominale (40). Il semble que l'efficacité de la CPAP soit dépendante de la durée des séances. Une CPAP nasale continue $(12-24 \mathrm{~h} / \mathrm{j})$ post opératoire comparée à des séances 
intermittentes (10 minutes/4h) diminue la durée de séjour en post opératoire de chirurgie aortique (41).

La VNI peut délivrer deux niveaux de pression (aide inspiratoire et pression expiratoire positive) et porte alors le nom anglais de BiPAP (BiPhasic Positive airway Pressure). Elle assure une amélioration de l'hématose après gastroplastie (42). Elle permet également (et surtout) de réduire la durée de séjour après chirurgie thoracique (30).

Pour résumer, l'utilisation de la VNI permet de prévenir la survenue de CRP, bien que l'on manque de données pour en définir les modalités exactes (durée des séances, durée totale).

\subsubsection{Sonde naso-gastrique}

L'abandon de la mise en place systématique d'une sonde naso-gastrique (SNG) en chirurgie abdominale a permis de réduire l'incidence des complications respiratoires post opératoires $(43,44)$, sans pour autant retarder la reprise du transit. La pose d'une SNG doit donc être réservée aux patients présentant d'importants vomissements post opératoires, une intolérance orale ou une distension abdominale symptomatique.

\subsubsection{Programmes de soins}

Les stratégies ci-dessus peuvent s'intégrer dans des programmes de soins plus vastes, faisant appel aux différents soignants et intégrant d'autres mesures facilement réalisables comme les bains de bouche bi quotidiens à la chlorexidine, la prise des repas assis, une position proclive à $30^{\circ}$ ou encore une déambulation précoce avec une bonne analgésie (45)

Devant les données parfois contradictoires et l'absence de recommandation des sociétés savantes françaises, il parait utile de donner quelques pistes pouvant être applicables en pratique courante

- en pré opératoire :

- Repérer les patients les plus à risque de développer une CRP

- chirurgie cardio-thoracique, abdominale sus-mésocolique 
- chirurgie en urgence

- chirurgie de longue durée (> 3 heures)

- âge > 60 ans,

- $\mathrm{BPCO}$

- Insuffisance cardiaque chronique

- Absence d'autonomie

- Aucun examen complémentaire ne peut permettre de repérer ces patients

- Arrêt du tabac

- Spirométrie incitative et kinésithérapie pour les patients à risque

- En per opératoire :

- Eviter l'anesthésie générale dès que possible

○ Mettre en œuvre une ventilation protectrice : Vt $6-8 \mathrm{ml} / \mathrm{kg}$ de poids idéal, PEEP 6-8 cmH20, manœuvres de recrutement

- Analgésie péridurale thoracique ou locorégionale et systémique

- En post opératoire :

- Sonde naso-gastrique seulement si nécessaire

- Favoriser l'expansion pulmonaire : CPAP, VNI, si possible en continu

○ Spirométrie incitative chez le patient compliant

○ Kinésithérapie respiratoire

○ Déambulation précoce, repas assis, position proclive $30^{\circ}$

\section{Prise en charge des CRP}

Malgré l'amélioration des pratiques de dépistage des patients à risque et de prévention des CRP, il arrive encore fréquemment que la fonction respiratoire d'un patient se dégrade en post opératoire. Les mécanismes en cause peuvent être multiples : atélectasie, épanchement pleural, pneumopathie débutante... Et chacune de ces causes nécessite une prise en charge spécifique qu'il serait trop long de détailler. Néanmoins, il convient de garder à l'esprit qu'une dégradation respiratoire peut toujours être un mode possible de révélation d'une complication chirurgicale (lâchage d'anastomose digestive par exemple). 
En dehors de ces cas où une reprise chirurgicale en urgence s'impose, la prise en charge de ces CRP relève d'une prise en charge réanimatoire classique : oxygénothérapie, VNI, réintubation, prélèvements bactériologiques larges, bilan radiologique...

\section{Conclusion}

Les complications respiratoires post opératoires sont une problématique grave et encore insuffisamment connue. Leur prise en charge repose sur des données scientifiques insuffisantes, et nous ne disposons pas encore de recommandations françaises à ce sujet.

Dans l'attente, il faut probablement s'attacher à dépister en amont les patients les plus à risque et adopter une attitude active de prévention, basée sur la ventilation protectrice peropératoire, une analgésie optimale et les techniques d'expansion pulmonaire.

\section{Bibliographie}

1. Canet J, Gallart L, Gomar C, Paluzie G, Vallès J, Castillo J, et al., ARISCAT Group. Prediction of postoperative pulmonary complications in a population-based surgical cohort. Anesthesiology, 2010; 113:1338- 50.

2. Smetana GW, Lawrence VA, Cornell JE, American College of Physicians. Preoperative pulmonary risk stratification for noncardiothoracic surgery: systematic review for the American College of Physicians. Ann Intern Med, 2006; 144:581-95.

3. Kor DJ, Warner DO, Alsara A, Fernández-Pérez ER, Malinchoc M, Kashyap R, et al. Derivation and diagnostic accuracy of the surgical lung injury prediction model. Anesthesiology, 2011; 115:117-28.

4. Arozullah AM, Daley J, Henderson WG, Khuri SF. Multifactorial Risk Index for Predicting Postoperative Respiratory Failure in Men After Major Noncardiac Surgery. Ann Surg, 2000; 232:242-53.

5. Manku K, Bacchetti P, Leung JM. Prognostic significance of postoperative in-hospital complications in elderly patients. I. Long-term survival. Anesth Analg, 2003; 96:583-9. 
6. Westbrook PR, Stubbs SE, Sessler AD, Rehder K, Hyatt RE. Effects of anesthesia and muscle paralysis on respiratory mechanics in normal man. J Appl Physiol, 1973; 34:816.

7. Hedenstierna G. Alveolar collapse and closure of airways: regular effects of anaesthesia. Clin Physiol Funct Imaging, 2003; 23:123-9.

8. Tokics L, Hedenstierna G, Strandberg A, Brismar B, Lundquist H. Lung collapse and gas exchange during general anesthesia: effects of spontaneous breathing, muscle paralysis, and positive end-expiratory pressure. Anesthesiology, 1987; 66:157-67.

9. Von Ungern-Sternberg BS, Regli A, Schneider MC, Kunz F, Reber A. Effect of obesity and site of surgery on perioperative lung volumes. Br J Anaesth, 2004; 92:202-7.

10. Burger EJ, Macklem P. Airway closure: demonstration by breathing 100 percent $\mathrm{O} 2$ at low lung volumes and by N2 washout. J Appl Physiol, 1968; 25:139-48.

11. Wollmer P, Schairer W, Bos JA, Bakker W, Krenning EP, Lachmann B. Pulmonary clearance of 99mTc-DTPA during halothane anaesthesia. Acta Anaesthesiol Scand, 1990; 34:572-5.

12. Mathru M, Esch O, Lang J, Herbert ME, Chaljub G, Goodacre B, et al. Magnetic resonance imaging of the upper airway. Effects of propofol anesthesia and nasal continuous positive airway pressure in humans. Anesthesiology, 1996; 84:273-9.

13. Sundman E, Witt H, Sandin R, Kuylenstierna R, Bodén K, Ekberg O, et al. Pharyngeal Function and Airway Protection During Subhypnotic Concentrations of Propofol, Isoflurane, and Sevoflurane: Volunteers Examined by Pharyngeal Videoradiography and Simultaneous Manometry. Anesthesiology, 2001; 95:1125-32.

14. Sundman E, Witt H, Olsson R, Ekberg O, Kuylenstierna R, Eriksson LI. The Incidence and Mechanisms of Pharyngeal and Upper Esophageal Dysfunction in Partially Paralyzed Humans: Pharyngeal Videoradiography and Simultaneous Manometry after Atracurium. Anesthesiology, 2000; 92:977-84.

15. Brown SR, Goodfellow PB. Transverse verses midline incisions for abdominal surgery. Cochrane Database Syst Rev, 2005; (4):CD005199.

16. Joris J, Kaba A, Lamy M. Postoperative spirometry after laparoscopy for lower abdominal or upper abdominal surgical procedures. Br J Anaesth, 1997; 79:422-6.

17. Joris JL, Hinque VL, Laurent PE, Desaive CJ, Lamy ML. Pulmonary function and pain after gastroplasty performed via laparotomy or laparoscopy in morbidly obese patients. Br J Anaesth, 1998; 80:283-8.

18. Warner DO. Preventing postoperative pulmonary complications: the role of the anesthesiologist. Anesthesiology, 2000; 92:1467-72. 
19. Ford GT, Grant DA, Rideout KS, Davison JS, Whitelaw WA. Inhibition of breathing associated with gallbladder stimulation in dogs. J Appl Physiol Bethesda Md 1984, 1988; 65:72-9.

20. Nunn JF. Effects of anaesthesia on respiration. Br J Anaesth, 1990; 65:54-62.

21. Arozullah AM, Khuri SF, Henderson WG, Daley J, Participants in the National Veterans Affairs Surgical Quality Improvement Program. Development and validation of a multifactorial risk index for predicting postoperative pneumonia after major noncardiac surgery. Ann Intern Med, 2001; 135:847-57.

22. Goldman L, Caldera DL, Nussbaum SR, Southwick FS, Krogstad D, Murray B, et al. Multifactorial Index of Cardiac Risk in Noncardiac Surgical Procedures. N Engl J Med, $1977 ; 297: 845-50$.

23. Lee TH, Marcantonio ER, Mangione CM, Thomas EJ, Polanczyk CA, Cook EF, et al. Derivation and Prospective Validation of a Simple Index for Prediction of Cardiac Risk of Major Noncardiac Surgery. Circulation, 1999; 100:1043-9.

24. Hua M, Brady JE, Li G. A scoring system to predict unplanned intubation in patients having undergone major surgical procedures. Anesth Analg, 2012; 115:88-94.

25. Mazo V, Sabaté S, Canet J, Gallart L, de Abreu MG, Belda J, et al. Prospective external validation of a predictive score for postoperative pulmonary complications. Anesthesiology, 2014; 121:219-31.

26. SFAR - Examens pré-interventionnels systématiques (RFE 2012) [Internet]. Disponible sur: http://www.sfar.org/article/901/examens-pre-interventionnels-systematiques-rfe2012

27. Qaseem A. Risk Assessment for and Strategies To Reduce Perioperative Pulmonary Complications for Patients Undergoing Noncardiothoracic Surgery: A Guideline from the American College of Physicians. Ann Intern Med, 2006; 144:575.

28. SFAR - Tabagisme périopératoire (CE 2005) [Internet].Disponible sur: http://www.sfar.org/article/151/tabagisme-perioperatoire-ce-2005

29. Lacasse Y, Goldstein R, Lasserson TJ, Martin S. Pulmonary rehabilitation for chronic obstructive pulmonary disease. Cochrane Database Syst Rev, 2006; (4):CD003793.

30. Perrin C, Jullien V, Vénissac N, Berthier F, Padovani B, Guillot F, et al. Prophylactic use of noninvasive ventilation in patients undergoing lung resectional surgery. Respir Med, 2007; 101:1572-8.

31. Ventilation with Lower Tidal Volumes as Compared with Traditional Tidal Volumes for Acute Lung Injury. N Engl J Med, 2000; 343:812-4.

32. Parsons PE, Eisner MD, Thompson BT, Matthay MA, Ancukiewicz M, Bernard GR, et al., NHLBI Acute Respiratory Distress Syndrome Clinical Trials Network. Lower tidal volume ventilation and plasma cytokine markers of inflammation in patients with acute lung injury. Crit Care Med, 2005; 33:1-6; discussion 230-2. 
33. Jaber S, Coisel Y, Chanques G, Futier E, Constantin J-M, Michelet P, et al. A multicentre observational study of intra-operative ventilatory management during general anaesthesia: tidal volumes and relation to body weight. Anaesthesia, 2012; 67:999-1008.

34. Hess DR, Kondili D, Burns E, Bittner EA, Schmidt UH. A 5-year observational study of lung-protective ventilation in the operating room: a single-center experience. J Crit Care, 2013; 28:533.e9-15.

35. Futier E, Constantin J-M, Paugam-Burtz C, Pascal J, Eurin M, Neuschwander A, et al., IMPROVE Study Group. A trial of intraoperative low-tidal-volume ventilation in abdominal surgery. N Engl J Med, 2013; 369:428-37.

36. Pöpping DM, Elia N, Marret E, Remy C, Tramèr MR. Protective effects of epidural analgesia on pulmonary complications after abdominal and thoracic surgery: a metaanalysis. Arch Surg Chic Ill 1960, 2008; 143:990-9; discussion 1000.

37. Beaussier M, El'ayoubi H, Rollin M, Parc Y, Atchabahian A, Chanques G, et al. Parietal analgesia decreases postoperative diaphragm dysfunction induced by abdominal surgery: a physiologic study. Reg Anesth Pain Med, 2009; 34:393-7.

38. Lawrence VA, Cornell JE, Smetana GW, American College of Physicians. Strategies to reduce postoperative pulmonary complications after noncardiothoracic surgery: systematic review for the American College of Physicians. Ann Intern Med, 2006; 144:596-608.

39. Freitas ERFS, Soares BGO, Cardoso JR, Atallah ÁN. Incentive spirometry for preventing pulmonary complications after coronary artery bypass graft. Cochrane Database Syst Rev, 2012; 9:CD004466.

40. Ferreyra GP, Baussano I, Squadrone V, Richiardi L, Marchiaro G, Del Sorbo L, et al. Continuous positive airway pressure for treatment of respiratory complications after abdominal surgery: a systematic review and meta-analysis. Ann Surg, 2008; 247:617-26.

41. Kindgen-Milles D, Müller E, Buhl R, Böhner H, Ritter D, Sandmann W, et al. Nasalcontinuous positive airway pressure reduces pulmonary morbidity and length of hospital stay following thoracoabdominal aortic surgery. Chest, 2005; 128:821-8.

42. Joris JL, Sottiaux TM, Chiche JD, Desaive CJ, Lamy ML. Effect of bi-level positive airway pressure (BiPAP) nasal ventilation on the postoperative pulmonary restrictive syndrome in obese patients undergoing gastroplasty. Chest, 1997; 111:665-70.

43. Nelson R, Tse B, Edwards S. Systematic review of prophylactic nasogastric decompression after abdominal operations. Br J Surg, 2005; 92:673-80.

44. Nelson R, Edwards S, Tse B. Prophylactic nasogastric decompression after abdominal surgery. Cochrane Database Syst Rev, 2007; (3):CD004929.

45. Wren SM, Martin M, Yoon JK, Bech F. Postoperative pneumonia-prevention program for the inpatient surgical ward. J Am Coll Surg, 2010; 210:491-5. 
